\title{
ALGEBRAIC KAHN-PRIDDY THEOREM
}

\author{
WEN-HSIUNG LIN
}

\begin{abstract}
There is an epimorphism from the stable homotopy of the infinite real projective space $R P^{\infty}$ to the (2-component) stable homotopy of spheres. This is the well known KahnPriddy theorem and was originally a conjecture of $M$. E. Mahowald and G. W. Whitehead. Mahowald also conjectured that the epimorphism should occur in the $E_{2}$ terms of the Adams spectral sequences. We prove this conjecture is true.
\end{abstract}

1. Introduction. In his memoir [3] M. E. Mahowald made two conjectures on a specific map $\lambda$ from the suspension spectrum $P^{\infty}$ of the infinite real projective space $R P^{\infty}$ to the sphere spectrum $S^{0}$. He conjectured that $\lambda$ induces epimorphisms in homotopy and in $E_{2}$ terms of the mod 2 Adams spectral sequences which are Ext groups over the mod 2 Steenrod algebra $A$. The first conjecture, which was also conjectured by G. W. Whitehead [5], has been proved by D. S. Kahn and S. B. Priddy [2] and is now known as the Kahn-Priddy theorem. The second conjecture, however, remains unproved. In this paper we record a proof of the truth of Mahowald's conjecture on this "algebraic Kahn-Priddy theorem". The result is stated as Theorem 1.1 below.

The map $\lambda$ cited above has the property that $\lambda_{\ddagger}: \pi_{1}\left(P^{\infty}\right)=Z_{2} \rightarrow$ $\pi_{1}\left(S^{\circ}\right)=Z_{2}$ is an isomorphism. Kahn and Priddy proved their theorem not just for $\lambda$ but also for any map $g: P^{\infty} \rightarrow S^{0}$ which induces isomorphism in $\pi_{1}(\quad)$. We shall state our "algebraic Kahn-Priddy theorem" also for any such map $g$.

TheOREM 1.1. For any map $g: P^{\infty} \rightarrow S^{0}$ that induces an isomorphism in the first stem homotopy groups the induced homomorphism

$$
g_{*}: \operatorname{Ext}_{A}^{s, t}\left(H^{*}\left(P^{\infty}\right), Z_{2}\right) \longrightarrow \operatorname{Ext}_{A}^{s+1, t+1}\left(H^{*}\left(S^{0}\right), Z_{2}\right)
$$

is an epimorphism for all $s$ and $t$ with $t-s \geqq 1$.

Here $H^{*}()$ is the reduced $\bmod 2$ cohomology functor. The result in Theorem 1.1 is what Mahowald had conjectured in [3] for $g=\lambda$. We shall see that only the isomorphism $g_{\sharp}: \pi_{1}\left(P^{\infty}\right) \rightarrow \pi_{1}\left(S^{0}\right)$ is relevent. The map $\lambda$ does not play a special role.

I would like to thank Professor Mahowald for encouragement to prove his conjecture.

2. Proof. The main result used to prove Theorem 1.1 is 
Proposition 2.1 which will be stated at the end of this section and proved in $\S 3$.

We first briefly describe the induced homomorphism $g_{*}$ in Theorem 1.1. Recall that $H^{*}\left(P^{\infty}\right)=\overline{Z_{2}[x]}$ with $\operatorname{dim}(x)=1$. The $\bmod 2$ Steenrod algebra acts on $H^{*}\left(P^{\infty}\right)$ by $S q^{i} x^{k}=\left(\begin{array}{l}k \\ i\end{array}\right) x^{k+i}$. Consider the following diagram consisting of the first stage of the mod 2 Adams resolution of $S^{0}$ and the map $g$ :

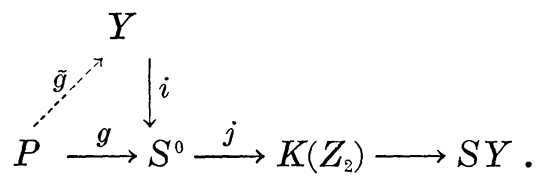

It is easy to see that $H^{*}(Y)=\Sigma^{-1} \bar{A}$ where $\bar{A}$ is the augmentation ideal of $A$ and we have the short exact sequence of $A$-modules

$$
\begin{gathered}
0 \longrightarrow H^{*}(S Y)=\bar{A} \longrightarrow H^{*}\left(K\left(Z_{2}\right)\right)=A \\
\stackrel{j^{*}}{\longrightarrow} H^{*}\left(S^{0}\right)=Z_{2} \longrightarrow 0 .
\end{gathered}
$$

Since $H^{0}\left(P^{\infty}\right)=0, j g: P^{\infty} \rightarrow K\left(Z_{2}\right)$ is null homotopic; so $g$ can be lifted to a map $\widetilde{g}: P^{\infty} \rightarrow Y$ as indicated in the diagram above. Composing the induced homomorphism

$$
\begin{gathered}
\left(\widetilde{g}^{*}\right)^{*}: \operatorname{Ext}_{A}^{s, t}\left(H^{*}\left(P^{\infty}\right), Z_{2}\right) \longrightarrow \operatorname{Ext}_{A}^{s, t}\left(H^{*}(Y), Z_{2}\right) \\
=\operatorname{Ext}_{A}^{s, t+1}\left(H^{*}(S Y), Z_{2}\right)
\end{gathered}
$$

with the coboundary homomorphism

$$
\delta^{*}: \operatorname{Ext}_{A}^{s, t+1}\left(H^{*}(S Y), Z_{2}\right) \longrightarrow \operatorname{Ext}_{A}^{s+1, t+1}\left(H^{*}\left(S^{0}\right), Z_{2}\right)
$$

which is obtained by applying $\operatorname{Ext}_{A}^{* * *}\left(, Z_{2}\right)$ to the short exact sequence (1) we get the homomorphism $g_{*}$ in Theorem 1.1.

The $A$-map $\widetilde{g}^{*}: H^{*}(Y)=\Sigma^{-1} \bar{A} \rightarrow H^{*}\left(P^{\infty}\right)$ is easy to describe. Recall that $\pi_{1}\left(S^{0}\right)=Z_{2}$ is generated by $\eta$ and $\eta$ is detected by $S q^{2}$. It is easy to infer from the isomorphism $g_{n}: \pi_{1}\left(P^{\infty}\right)=Z_{2} \rightarrow$ $\pi_{1}\left(S^{0}\right)=Z_{2}$ that $\widetilde{g}^{*}\left(S q^{2}\right)=x$ and then from the $A$-module structure of $H^{*}\left(P^{\infty}\right)$ that $\widetilde{g}^{*}\left(S q^{i}\right)=x^{i-1}$ for all $i \geqq 2$. It is clear that $\widetilde{g}^{*}\left(S q^{1}\right)=$ 0 . Thus $\widetilde{g}^{*}$ is independent of the lifting $\widetilde{g}$ and also independent of $g: P^{\infty} \rightarrow S^{0}$ for which $g$ : is an isomorphism in $\pi_{1}(\quad)$.

We next recall ([1]) that for any locally finite left $A$-module $M$ a suitable small complex for computing $\operatorname{Ext}_{1}^{* * *}\left(M, Z_{2}\right)$ is $M^{*} \otimes \Lambda$ where $M^{*}$ is the dual $Z_{2}$-module of $M$ and $\Lambda$ is the lamda algebra. $\Lambda$ is a bigraded differential algebra over $Z_{2}$ generated by $\lambda_{i}(i \geqq 0)$ with $\lambda_{i} \in 1^{1, i+1}$ subject to the following relations 


$$
\lambda_{i} \lambda_{2 i+1+n}=\sum_{j \geq 0}\left(\begin{array}{c}
n-j-1 \\
j
\end{array}\right) \lambda_{i+n-j} \lambda_{2 i+1+j} ;
$$

the differential $\delta$ is given by

$$
\delta\left(\lambda_{k}\right)=\sum_{j \geq 0}\left(\begin{array}{c}
k-j-1 \\
j+1
\end{array}\right) \lambda_{k-j-1} \lambda_{j} .
$$

$M^{*} \oplus \Lambda$ is a differential right $\Lambda$-module with differential $\delta$ given by

$$
\delta\left(m^{*}\right)=\sum_{i \geq 0} m^{*} S q^{i+1} \otimes \lambda_{i}
$$

where the right $A$-module structure of $M^{*}$ is obtained by transposing the left $A$-module structure of $M$.

In particular $H^{*},{ }^{*}(\Lambda)=\operatorname{Ext}_{A}^{*, *}\left(Z_{2}, Z_{2}\right)=\operatorname{Ext}_{A}^{*, *}\left(H^{*}\left(S^{0}\right), Z_{2}\right)$ and $H^{*, *}\left(H_{*}\left(P^{\infty}\right) \otimes \Lambda\right)=\operatorname{Ext}_{A}^{*, *}\left(H^{*}\left(P^{\infty}\right), Z_{2}\right)$ where $H_{*}\left(P^{\infty}\right)=\left(H^{*}\left(P^{\infty}\right)\right)^{*}$ is the mod 2 homology of $P^{\infty}$. Let $\left\{y_{k}\right\}_{k \geq 1}$ be the $Z_{2}$-base of $H_{*}\left(P^{\infty}\right)$ dual to $\left\{x^{k}\right\}_{k \geq 1}$. The differential $\delta$ of $H_{*}\left(P^{\infty}\right) \otimes \Lambda$ is given by

$$
\delta\left(y_{k}\right)=\sum_{j \geq 0}\left(\begin{array}{c}
k-j-1 \\
j+1
\end{array}\right) y_{k-j-1} \otimes \lambda_{j} .
$$

The $A$-map $\widetilde{g}^{*}: H^{*}(Y) \rightarrow H^{*}\left(P^{\infty}\right)$ gives rise to a chain map $\phi$ : $H_{*}\left(P^{\infty}\right) \otimes \Lambda \rightarrow \Lambda$ which induces the homomorphism $g_{*}$ in Theorem 1.1. The chain map $\phi$ is a differential $\Lambda$-map and on the generators $y_{k}$ it is given by

$$
\phi\left(y_{k}\right)=\lambda_{k} .
$$

Our method to prove Theorem 1.1 is to construct a chain map $\psi: \Lambda \rightarrow H_{*}\left(P^{\infty}\right) \otimes \Lambda$ (which is not a differential $\Lambda$-map) so that the composite $\Phi=\phi \psi: \Lambda \rightarrow \Lambda$ has the property that for any pair of integers $(s, t)$ with $t-s \geqq 1$ there is an integer $m=m(s, t) \geqq 1$ such that the iterated product $\Phi^{m}$ is equal to the identity map on $\Lambda^{s, t}$.

To construct $\psi$ we define some terminology and fix some notations. From the relations (2) we see a $Z_{2}$-base for $\Lambda^{s}\left(\Lambda^{s}=\sum_{t} \Lambda^{s, t}\right)$ is given by $\left\{\lambda_{i_{1}} \lambda_{i_{2}} \cdots \lambda_{i_{s}} \mid 2 i_{1} \geqq i_{2}, \cdots, 2 i_{s-1} \geqq i_{s}\right\}$. Any such monomial $\lambda_{i_{1}} \lambda_{i_{2}} \cdots \lambda_{i_{s}}$ is called an admissible monomial and the corresponding sequence $\left(i_{1}, i_{2}, \cdots, i_{s}\right)$ is called an admissible sequence. If $J=$ $\left(j_{1}, j_{2}, \cdots, j_{s}\right)$ is a sequence of nonnegative integers then we write $\lambda_{J}$ to denote $\lambda_{j_{1}} \lambda_{j_{2}} \cdots \lambda_{j_{s}}$. If $I=\left(i_{1}, i_{2}, \cdots, i_{s}\right)$ is an admissible sequence and $J=\left(j_{1}, j_{2}, \cdots, j_{s}\right)$ is an sequence of nonnegative 
integers (whether it is admissible or not) then we write $\lambda_{I} \in \lambda_{J}$ to mean that $\lambda_{I}$ appears in the admissible expansion of $\lambda_{J}$.

We construct $\psi^{s}: \Lambda^{s} \rightarrow H_{*}\left(P^{\infty}\right) \otimes \Lambda^{s}$ as follows. $\psi^{0}(1)=0, \psi^{1}\left(\lambda_{0}\right)=$ 0 and $\psi^{1}\left(\lambda_{i}\right)=y_{i} \otimes 1$ for $i \geqq 1$. For $s \geqq 2$ it is a little complicated to describe $\psi^{s}$. Let $\lambda_{I}=\lambda_{i_{1}} \lambda_{i_{2}} \cdots \lambda_{i_{s}}$ be a given admissible monomial in $\Lambda^{s}\left(s \geqq 2\right.$ ) and let $I^{\prime}=\left(i_{2}, \cdots, i_{s}\right)$. If $i_{1}=0$ (which implies $i_{\mu}=0$ for all $\mu \geqq 2$ ) then define $\psi^{s}\left(\lambda_{I}\right)=0$. If $i_{1}>0$ then $\psi^{s}\left(\lambda_{I}\right)$ will be of the form

$$
y_{i_{1}} \otimes \lambda_{I^{\prime}}+\sum_{\nu} y_{j_{1}(\nu)} \otimes \lambda_{J^{\prime}(\nu)}
$$

where the second sum is described as follows. First of all we require that each $\lambda_{J^{\prime}(2)}$ be an admissible monomial in $\Lambda^{s-1}$ and that $J(\nu)=\left(j_{1}(\nu), j_{2}(\nu), \cdots, j_{s}(\nu)\right)$, where $J^{\prime}(\nu)=\left(j_{2}(\nu), \cdots, j_{s}(\nu)\right)$, be inadmissible. Secondly, choose any positive integer $m$ so that $m \gg i_{1}+$ $i_{2}+\cdots+i_{s}+s$ (which means $m$ is very large compared with the integers $i_{j}$ and $s$ ) and consider the sequence $J(\nu, m)=\left(2^{m}+j_{1}(\nu)\right.$, $\left.j_{2}(\nu), \cdots, j_{s}(\nu)\right)$. It is clear that $J(\nu, m)$ is admissible. Then $y_{j_{1}(\nu)} \otimes$ $\lambda_{J^{\prime}(\nu)}$ occurs in the second sum of (3) if and only if $\lambda_{J(\nu, m)} \in \lambda_{I(q, m)}$ for some $q$ with $2 \leqq q \leqq s$ where $I(q, m)=\left(i_{1}, \cdots, i_{q}+2^{m}, i_{q+1}, \cdots, i_{s}\right)$. We shall see later that the second sum of (3) is independent of the large positive integer $m$.

Consider the composite $\Phi^{s}=\phi^{s} \psi^{s}: \Lambda^{s} \rightarrow \Lambda^{s}$. It is clear that $\Phi^{0}(1)=$ $0, \Phi^{1}\left(\lambda_{0}\right)=0$ and $\Phi^{1}\left(\lambda_{2}\right)=\lambda_{i}$ for $i \geqq 1$. For $s \geqq 2$ let $I=\left(i_{1}, i_{2}, \cdots, i_{s}\right)$ be a given admissible sequence. If $i_{1}=0$ then $\Phi^{s}\left(\lambda_{I}\right)=0$. If $i_{i}>0$ and if we still use the notation in the preceding paragraph then

$$
\Phi^{s}\left(\lambda_{I}\right)=\lambda_{I}+\sum_{\nu} \lambda_{J(\nu)}
$$

Note that each $\lambda_{J(\nu)}$ is an inadmissible monomial. Suppose $\sum_{\nu} \lambda_{J(\nu)} \neq$ 0 and let $\sum_{/ l} \lambda_{I(\mu)}$ be the admissible expansion of $\sum_{\nu} \lambda_{J(\nu)}$; so

$$
\Phi^{s}\left(\lambda_{I}\right)=\lambda_{I}+\sum_{\mu} \lambda_{I(\mu)} .
$$

Proposition 2.1. ( i ) $\psi^{*}: \Lambda^{*} \rightarrow H^{*}\left(P^{\infty}\right) \otimes \Lambda^{*}$ is a chain map (so $\Phi^{*}: \Lambda^{*} \rightarrow \Lambda^{*}$ is also a chain $\operatorname{map}$ ).

(ii) For $s \geqq 2$ let $\lambda_{I}$ be any admissible monomial in $\Lambda^{s}$ such that the first entry of $I$ is positive. Suppose $\Phi^{s}\left(\lambda_{I}\right)$ is as described in (4). Then the first entry of $I$ is strictly less than that of each $I(\mu)$.

It follows from Proposition 2.1 that $\Phi$ has the property described above, that is, for any pair of integers $(s, t)$ with $t-s \geqq 1$ there is some integer $m=m(s, t) \geqq 1$ such that $\Phi^{m}$ is equal to the identity 
map on $\Lambda^{s, t}$. Since $\psi^{*}: H_{*}\left(P^{\infty}\right) \otimes \Lambda^{*} \rightarrow \Lambda^{*}$ induces the homomorphism $g_{*}$ in Theorem 1.1 the result in the theorem follows.

3. Proof of Proposition 2.1. We begin with the proof of Proposition 2.1 (ii) which is easier than that of Proposition 2.1 (i). To prove Proposition 2.1 (ii) we begin with some lemmas which will also be needed in the proof of Proposition 2.1 (i).

LEMMA 3.1. Let $I=\left(i_{1}, \cdots, i_{n}\right)$ be an admissible sequence $(n \geqq 2)$. Suppose $J=\left(j_{1}, \cdots, j_{n}\right)$ is a sequence such that $\lambda_{I} \in \lambda_{J}$. Then

(i ) $i_{1} \leqq m(J)$ where $m(J)=\max _{l}\left\{j_{l}\right\}$ and (ii) $j_{1} \leqq i_{1}$.

The proof is given by induction on the lexicographical order from the right of the sequence $J=\left(j_{1}, \cdots, j_{n}\right)$, the first inductive step being the case $\left(j_{1}, \cdots, j_{n}\right)=\left(i_{1}, \cdots, i_{n}\right)$. We leave the details of the proof to the reader.

LEMMA 3.2. Let $\left(j_{1}, \cdots, j_{s}\right)$ be an admissible sequence ( $\left.s \geqq 2\right)$ and $m$ be a positive integer such that $m \gg j_{1}+\cdots+j_{s}+s$. Suppose $\left(p_{1}, \cdots, p_{s}\right)$ is a sequence of nonnegative integers such that $\left(p_{2}, \cdots, p_{s}\right)$ is admissible and

$$
\lambda_{2^{m}+p_{1}} \lambda_{p_{2}} \cdots \lambda_{p_{s}} \in \lambda_{j_{1}} \cdots \lambda_{j_{k-1}} \lambda_{2^{m}+j_{k}} \lambda_{j_{k+1}} \cdots \lambda_{j_{s}}
$$

for some $k$ with $2 \leqq k \leqq s+1$. Then (i) $p_{2} \geqq 2 j_{1}+1$ and (ii) $\left(p_{1}, p_{2}, \cdots, p_{s}\right)$ is inadmissible, i.e., $2 p_{1}<p_{2}$.

For the proof of Proposition 2.1 (ii) we shall only need the result in Lemma 3.2 (i).

Proof of Lemma 3.2. In our proof there will arise variables $\nu_{k-1}, \nu_{k-2}, \cdots, \nu_{1}$ with integral values and integers

$$
\begin{aligned}
& 2^{m}+j_{k}-j_{k-1}-\cdots-j_{k-t+1}-2 j_{k-t}-\nu_{k-1}-\nu_{k-2}-\cdots-\nu_{k-t}-t-1, \\
& 2^{m}+j_{k}-j_{k-1}-\cdots-j_{k-t+1}-j_{k-t}-\nu_{k-1}-\nu_{k-2}-\cdots-\nu_{k-t}-t
\end{aligned}
$$

and

$$
2 j_{k-t}+\nu_{k-t}+1
$$

where $1 \leqq t \leqq k-1$. We denote these integers by $\mu_{k-t}, j_{t}^{\prime \prime}$ and $j_{t}^{\prime}$ in that order.

Since $m$ is large and $k \geqq 2,\left(j_{k-1}, 2^{m}+j_{k}\right)$ is inadmissible. So we can expand $\lambda_{j_{k-1}} \lambda_{2}{ }^{m+j_{k}}$ into sum of admissible monomials by using the relations (2). We have 


$$
\begin{aligned}
& \lambda_{j_{1}} \cdots \lambda_{j_{k-2}} \lambda_{j_{k-1}} \lambda_{2} m+j_{k} \lambda_{j_{k+1}} \cdots \lambda_{j_{s}} \\
& \quad=\sum_{\nu_{k-1} \geqq 0}\left(\begin{array}{c}
\mu_{k-1} \\
\nu_{k-1}
\end{array}\right) \lambda_{j_{1}} \cdots \lambda_{j_{k-2}} \lambda_{j_{1}^{\prime \prime}} \lambda_{j_{1}^{\prime}} \lambda_{j_{k+1}} \cdots \lambda_{j_{s}} .
\end{aligned}
$$

From the assumption in the lemma we see that there is at least one $\nu_{k-1}$ for which

$$
\left(\begin{array}{l}
\mu_{k-1} \\
\nu_{k-1}
\end{array}\right) \equiv 1(\bmod 2)
$$

and

$$
\lambda_{2} m+p_{1} \lambda_{p_{2}} \cdots \lambda_{p_{s}} \in \lambda_{j_{1}} \cdots \lambda_{j_{k-2}} \lambda_{j_{1}^{\prime}} \lambda_{j_{1}} \lambda_{j_{k+1}} \cdots \lambda_{j_{s}}
$$

By Lemma 3.1 (i) either

$$
j_{1}^{\prime \prime}=2^{m}+j_{k}-j_{k-1}-\nu_{k-1}-1 \geqq 2^{m}+p_{1}
$$

or

$$
j_{1}^{\prime}=2 j_{k-1}+\nu_{k-1}+1 \geqq 2^{m}+p_{1}
$$

(since $m$ is large). If $2 j_{k-1}+\nu_{k-1}+1 \geqq 2^{m}+p_{1}$ then $\nu_{k-1} \gg \mu_{k-1}=$ $2^{m}+j_{k}-2 j_{k-1}-\nu_{k-1}-2$ and we would have $\left(\begin{array}{l}\mu_{k-1} \\ \nu_{k-1}\end{array}\right) \equiv 0(\bmod 2)$. Therefore $j_{1}^{\prime \prime} \geqq 2^{m}+p_{1}$. Thus $\left(j_{k-2}, j_{1}^{\prime \prime}\right)$ is admissible (if $k>2$ ). By expanding $\lambda_{j_{k-2}} \lambda_{j_{1}^{\prime \prime}}$ we find

$$
\begin{aligned}
& \lambda_{j_{1}} \cdots \lambda_{j_{k-3}} \lambda_{j_{k-2}} \lambda_{j_{1}^{\prime}} \lambda_{j_{1}^{\prime}} \lambda_{j_{k+1}} \cdots \lambda_{j_{s}} \\
& \quad=\sum_{\nu_{k-2} \geqq 0}\left(\begin{array}{c}
\mu_{k-2} \\
\nu_{k-2}
\end{array}\right) \lambda_{j_{1}} \cdots \lambda_{j_{k-3}} \lambda_{j_{2}^{\prime \prime}} \lambda_{j_{2}^{\prime}} \lambda_{j_{1}^{\prime}} \cdots \lambda_{j_{s}} .
\end{aligned}
$$

Hence there is at least one $\nu_{k-2}$ for which

$$
\left(\begin{array}{l}
\mu_{k-2} \\
\nu_{k-2}
\end{array}\right) \equiv 1(\bmod 2)
$$

and

$$
\lambda_{2}{ }^{m+p_{1}} \lambda_{p_{2}} \cdots \lambda_{p_{s}} \in \lambda_{j_{1}} \cdots \lambda_{j_{k-3}} \lambda_{j_{2}^{\prime}} \lambda_{j_{2}^{\prime}} \lambda_{j_{1}^{\prime}} \cdots \lambda_{j_{s}}
$$

Again from Lemma 3.1 (i) and the fact $\left(\begin{array}{l}\mu_{k-2} \\ \nu_{k-2}\end{array}\right) \equiv 1(\bmod 2)$ we must have $j_{2}^{\prime \prime} \geqq 2^{m}+p_{1}$. Thus $\left(j_{k-3}, j_{2}^{\prime \prime}\right)$ is inadmissible and we can continue to expand $\lambda_{j_{k-3}} \lambda_{j_{2}^{\prime \prime}}$ (if $k>3$ ). Continue this argument a finite number of times we end up with

$$
\lambda_{2 m+p_{1}} \lambda_{p_{2}} \cdots \lambda_{p_{s}} \in \lambda_{j_{k^{\prime}-1}^{\prime}} \lambda_{j_{k-1}^{\prime}} \cdots \lambda_{j_{2}^{\prime}} \lambda_{j_{1}^{\prime}} \cdots \lambda_{j_{s}}
$$

for some values of $\nu_{1}, \cdots, \nu_{k-1}$ which are nonegative and we have 
$j_{k-1}^{\prime \prime} \geqq 2^{m}+p_{1}$. Since $m$ is large, $2^{m}+p_{1}$ is actually equal to $j_{k-1}^{\prime \prime}$ i.e.,

$$
2^{m}+p_{1}=2^{m}+j_{k}-j_{k-1}-\cdots-j_{1}-\nu_{k-1}-\nu_{k-2}-\cdots-\nu_{1}-k+1
$$

or

$$
p_{1}=j_{k}-j_{k-1}-\cdots-j_{1}-\nu_{k-1}-\nu_{k-2}-\cdots-\nu_{1}-k+1
$$

and

$$
\lambda_{p_{2}} \cdots \lambda_{p_{s}} \in \lambda_{j_{k-1}^{\prime}} \cdots \lambda_{j_{2}} \lambda_{j_{1}^{\prime}} \cdots \lambda_{j_{s}} .
$$

By Lemma 3.1 (ii) $p_{2} \geqq j_{k-1}^{\prime}=2 j_{1}+\nu_{1}+1 \geqq 2 j_{1}+1$. This proves part (i) of the lemma. Since $\left(j_{1}, \cdots, j_{s}\right)$ is admissible, we have $2 j_{1} \geqq j_{2}, 2 j_{2} \geqq j_{3}, \cdots, 2 j_{k-1} \geqq j_{k}$ which imply $j_{1} \geqq j_{k} / 2^{k-1}, j_{2} \geqq j_{k} / 2^{k-2}, \cdots$, $j_{k-1} \geqq j_{k} / 2$. Then

$$
\begin{aligned}
p_{2}- & 2 p_{1} \geqq 2 j_{1}+1-2 j_{k}+2\left(j_{k-1}+\cdots+j_{1}\right)+2\left(\nu_{k-1}+\cdots+\nu_{1}\right) \\
& +2 k-2 \geqq 2\left(2 j_{1}+j_{2}+\cdots+j_{k-1}\right)-2 j_{k}+2 k-1 \\
\geqq & 2\left(1 / 2^{k-2}+1 / 2^{k-2}+1 / 2^{k-3}+\cdots+1 / 2\right) j_{k}-2 j_{k}+2 k-1 \\
= & 2 j_{k}-2 j_{k}+2 k-1=2 k-1>0,
\end{aligned}
$$

that is, $p_{2}>2 p_{1}$. This proves Lemma 3.1 (ii).

Proof of Proposition 2.1 (ii). We recall that

$$
\Phi^{s}\left(\lambda_{I}\right)=\lambda_{I}+\sum_{\nu} \lambda_{J(\nu)}
$$

and $\sum_{\mu} \lambda_{I(\mu)}$ is the admissible expansion of $\sum_{\nu} \lambda_{J(\nu)}$ where each $J(\nu)=\left(j_{1}(\nu), j_{2}(\nu), \cdots, j_{s}(\nu)\right)$ is inadmissible, $\left(j_{2}(\nu), \cdots, j_{s}(\nu)\right)$ is admissible and

$$
\lambda_{2^{m}+j_{1}(\nu)} \lambda_{j_{2}(\nu)}, \cdots, \lambda_{j_{s}(\nu)} \in \lambda_{i_{1}} \cdots \lambda_{i_{k-1}} \lambda_{2^{m}+i_{k}} \cdots \lambda_{i_{s}}
$$

for some $k$ with $2 \leqq k \leqq s$ ( $m$ is large). Let $I(\mu)=\left(i_{1}(\mu), \cdots, i_{s}(\mu)\right)$. We want to show that $i_{1}(\mu)>i_{1}$ for all $\mu$ where $I=\left(i_{1}, \cdots, i_{s}\right)$.

By Lemma 3.2 (i) we have

(a) $j_{2}(\nu) \geqq 2 i_{1}+1$ for all $\nu$.

We shall prove

(b) For each $\mu$ there is some $\nu$ such that

$$
2 i_{1}(\mu)+1>j_{2}(\nu) \text {. }
$$

It is clear that the desired result follows from (a) and (b).

We suppose $\mu$ is a given index element. Since $\sum_{\mu} \lambda_{I(\mu)}$ is the admissible expansion of $\sum_{\nu} \lambda_{J(\nu)}$ there is some $\nu$ such that

$$
\lambda_{i_{1}(\mu)} \cdots \lambda_{i_{s}(\mu)} \in \lambda_{j_{1}(\nu)} \lambda_{j_{2}(\nu)} \cdots \lambda_{j_{s}(\nu)}
$$


By assumption $\left(j_{1}(\nu), j_{2}(\nu)\right)$ is inadmissible; so we can expand $\lambda_{j_{1}(\nu)} \lambda_{j_{2}(\nu)}$ into sum of admissible monomials. We have

$$
\begin{aligned}
& \lambda_{j_{1}(\nu)} \lambda_{j_{2}(\nu)} \cdots \lambda_{j_{s}(\nu)} \\
& \quad=\sum_{r \geqq 0}\left(\begin{array}{c}
j_{2}(\nu)-2 j_{1}(\nu)-r-2 \\
r
\end{array}\right) \lambda_{j_{2}(\nu)-j_{1}(\nu)-r-1} \lambda_{2 j_{1}(\nu)+r+1} \cdots \lambda_{j_{s}(\nu)} .
\end{aligned}
$$

Hence there is some $r$ for which

$$
\left(\begin{array}{c}
j_{2}(\nu)-2 j_{1}(\nu)-r-2 \\
r
\end{array}\right) \equiv 1(\bmod 2)
$$

and

$$
\lambda_{i_{1}(\mu)} \cdots \lambda_{i_{i_{s}}(\mu)} \in \lambda_{j_{0}(\nu)-j_{1}(\nu)-r-1} \lambda_{2 j_{1}(\nu)+r+1} \cdots \lambda_{j_{s}(\nu)} .
$$

From (5) we see that

$$
\left(j_{2}(\nu)-2 j_{1}(\nu)-r-2\right)-r \geqq 0
$$

or

$$
j_{2}(\nu) / 2 \geqq j_{1}(\nu)+r+1 .
$$

From (6) and Lemma 3.1 (ii) we have

$$
\begin{aligned}
i_{1}(\mu) & \geqq j_{2}(\nu)-\left(j_{1}(\nu)+r+1\right) \\
& \geqq j_{2}(\nu)-j_{2}(\nu) / 2=j_{2}(\nu) / 2
\end{aligned}
$$

i.e.,

$$
\begin{aligned}
& 2 i_{1}(\mu) \geqq j_{2}(\nu) \text { which implies } \\
& 2 i_{1}(\mu)+1>j_{2}(\nu) .
\end{aligned}
$$

This proves (b) and therefore Proposition 2.1 (ii).

Our proof of Proposition 2.1 (i) is rather lengthy. The construction of the map $\psi^{*}: \Lambda^{*} \rightarrow H_{*}\left(P^{\infty}\right) \otimes \Lambda^{*}$ in $\S 2$ comes from considering the dual complex $\bar{K}_{*}(A)$ of $\Lambda^{*}$, called Koszul complex for the Steenrod algebra $A$, in the bar construction $\bar{B}_{*}(A)$ as described by S. B. Priddy in [4]. We shall prove Proposition 2.1 (i) by showing that the dual $Z_{2}$-map $\psi_{*}: \bar{K}_{*}(A) \otimes H^{*}\left(P^{\infty}\right) \rightarrow \bar{K}_{*}(A)$ of $\psi^{*}$ is a chain map (Proposition 3.6).

We will first describe the structure of $\bar{K}_{*}(A)$ and then summarize the main properties of $\bar{K}_{*}(A)$ in Theorem 3.3 below. $\bar{K}_{0}(A)=$ $\bar{B}_{0}(A)=Z_{2}$ and $\bar{K}_{1}(A)$ is the $Z_{2}$-submodule of $\bar{B}_{1}(A)=\bar{A}$ which has $\left\{S q^{i} \mid i \geqq 1\right\}$ as a $Z_{2}$-base. To describe $\bar{K}_{s}(A)$ for $s \geqq 2$ consider any admissible sequence $I=\left(i_{1}, \cdots, i_{s}\right)$. Let $\Gamma_{I}=\left[S q^{i_{s}+1}|\cdots| S q^{i_{1}+1}\right]+Q_{I}$ where $Q_{I}$ is the sum of all $\left[S q^{j_{s}+1}|\cdots| S q^{j_{1}+1}\right]$ such that $J=\left(j_{1}, \cdots, j_{s}\right)$ 
is inadmissible and $\lambda_{I} \in \lambda_{J}$. Then $\bar{K}_{s}(A)$ is the $Z_{2}$-submodule of $\bar{B}_{s}(A)$ which has $\left\{\Gamma_{I} \mid I=\left(i_{1}, \cdots, i_{s}\right)\right.$ is admissible $\}$ as a $Z_{2}$-base. For $s=2$ we can explicitly write out, from the relations (2) in $\S 2$, the terms in the sum $\Gamma_{I}=\Gamma_{\left(i_{1}, i_{2}\right)}$; we have

$$
\Gamma_{\left(i_{1}, i_{2}\right)}=\left[S q^{i_{2}+1} \mid S q^{i_{1}+1}\right]+\sum_{j=1}^{\left[i_{2}+1 / 2\right]}\left(\begin{array}{c}
i_{1}-j \\
i_{2}+1-2 j
\end{array}\right)\left[S q^{i_{1}+i_{2}+2-j} \mid S q^{j}\right]
$$

where $[x]$ denotes the greatest integer less than or equal to $x$. From the Adem relations in the Steenrod algebra $A$ we have

$$
\begin{aligned}
& \bar{d}\left(\Gamma_{\left(i_{1}, i_{2}\right)}\right) \\
& \quad=S q^{i_{2}+1} S q^{i_{1}+1}+\sum_{j=1}^{\left[i_{2}+1 / 2\right]}\left(\begin{array}{c}
i_{1}-j \\
i_{2}+1-2 j
\end{array}\right) S q^{i_{1}+i_{2}+2-j} S q^{j} \\
& =\left(\begin{array}{c}
i_{1} \\
i_{2}+1
\end{array}\right) S q^{i_{1}+i_{2}+2}
\end{aligned}
$$

where $\bar{d}$ is the boundary homomorphism of $\bar{B}_{*}(A)$.

THeOREM 3.3 ([4]). Let $M$ be a locally finite A-module, $\left\{m_{j}\right\} a$ $Z_{2}$-base of $M$ and $\left\{m_{j}^{*}\right\}$ the dual $Z_{2}$-base of $M^{*}$.

(i) $\bar{K}_{*}(A) \otimes M$ is a subcomplex of the bar construction $\bar{B}_{*}(A) \otimes$ $M$ and the inclusion map $i \otimes M: \bar{K}_{*}(A) \otimes M \rightarrow \bar{B}_{*}(A) \otimes M$ is a chain equivalence.

(ii) The complex $\bar{K}_{*}(A) \otimes M$ is dual to the complex $M^{*} \otimes \Lambda$ in the sense that $\left\{\Gamma_{I} \otimes m_{j} \mid I\right.$ is admissible $\}\left(\Gamma_{I}=S q^{i_{1}+1}\right.$ if $\left.I=\left(i_{1}\right)\right)$ is dual to the $Z_{2}$-base $\left\{m_{j}^{*} \otimes \lambda_{I} \mid I\right.$ is admissible $\}$ of $M^{*} \otimes \Lambda$ and that the boundary homomorphism of $\bar{K}_{*}(A) \otimes M$ is dual to the differential of $M^{*} \otimes \Lambda$.

(iii) For a homogeneous element $R=\sum_{j}\left[S q^{j_{1}}|\cdots| S q^{j_{s}}\right] \in \bar{B}_{s}(A)$ $(s \geqq 2)$ to be an element of $\bar{K}_{s}(A)$ it is necessary and sufficient that, for each $i(1 \leqq i \leqq s-1)$ and each pair of sequences $\left(k_{1}, \cdots, k_{i-1}\right)$ and $\left(k_{i+2}, \cdots, k_{s}\right)$, the following condition holds

$$
\sum_{j} S q^{j_{i}} S q^{j_{i+1}}=S q^{c} \quad \text { or } \quad 0
$$

where the summation is taken over all $j$ such that $\left(j_{1}, \cdots, j_{i-1}\right)=$ $\left(k_{1}, \cdots, k_{i-1}\right)$ and $\left(j_{i+2}, \cdots j_{s}\right)=\left(k_{i+2}, \cdots, k_{s}\right)$.

We should remark that in [4] the complex $\bar{K}_{*}(A)$ is approached from the Koszul resolution viewpoint and the property in Theorem 3.3 (iii) is taken as the definition of $\bar{K}_{*}(A)$. The $Z_{2}$-base of $\bar{K}_{*}(A)$ described above is not explicitly exhibited there.

The property in Theorem 3.3 (iii) will be important to the proof 
of Proposition 2.1 (i). From (7), (8) and the Adem relations one can easily prove Theorem 3.3 (iii) for $s=2$ and we would like to make the following remark.

REMARK 3.4. The condition " $\sum_{j} S q^{j_{i}} S q^{j_{i+1}}=S q^{c}$ or 0 " in Theorem 3.3 (iii), for $s>2$, can be replaced by the condition " $\sum_{j}\left[S q^{j_{i}} \mid S q^{j_{i+1}}\right] \in$ $\bar{K}_{2}(A)$ '.

We proceed to describe the map $\psi_{*}: \bar{K}_{*}(A) \otimes H^{*}\left(P^{\infty}\right) \rightarrow \bar{K}_{*}(A)$ dual to $\psi^{*}: \Lambda^{*} \rightarrow H_{*}\left(P^{\infty}\right) \otimes \Lambda^{*}$. It is clear that $\psi_{0}\left(1 \otimes x^{i}\right)=S q^{i+1}$. To describe $\psi_{s}$ for $s \geqq 1$ consider any basis element $\Gamma_{I^{\prime}} \otimes x^{i}$ of $\bar{K}_{s}(A) \otimes H^{*}\left(P^{\infty}\right)$ where $I^{\prime}=\left(i_{1}, \cdots, i_{s}\right)$. Let $I=\left(i, i_{1}, \cdots, i_{s}\right)$. Choose any positive integer $m$ such that $m \gg i+i_{1}+\cdots+i_{s}+s$ and let $I(m)=\left(2^{m}+i, i_{1}, \cdots, i_{s}\right)$. Since $m$ is large, $I(m)$ is an admissible sequence. Consider the corresponding basis element

$$
\Gamma_{I(m)}=\left[S q^{i_{s}+1}|\cdots| S q^{i_{1}+1} \mid S q^{2^{2}+i+1}\right]+Q_{I(m)} .
$$

Let us write $\left[S q^{j_{1}}|\cdots| S q^{j_{s+1}}\right] \in Q_{I(m)}$ to mean that $\left[S q^{j_{1}}|\cdots| S q^{j_{s+1}}\right]$ appears in the sum $Q_{I(m)}$. By Lemma 3.1 (i) for each $\left[S q^{j_{1}}|\cdots| S q^{j_{s+1}}\right] €$ $Q_{I(m)}$ there is at least one $k$ such that $j_{k} \geqq 2^{m}+i+1>2^{m}$ and since $m$ is large there is only one such $k$. So each $\left[S q^{j_{1}}|\cdots| S q^{j_{s+1}}\right] \in$ $\Gamma_{I(m)}$ has exactly one $j_{k}>2^{m}$. We construct an element $\Omega_{I} \in \bar{B}_{s+1}(A)$ as follows. For each $\left[S q^{j_{1}}|\cdots| S q^{j_{s+1}}\right] \in \Gamma_{I(m)}$ let $j_{k}$ be the only integer such that $j_{k}>2^{m}$ and consider $\left[S q^{j_{1}^{\prime}}|\cdots| S q_{s+1}^{j^{\prime}}\right]$ where $j_{l}^{\prime}=j_{l}$ if $l \neq k$ and $j_{k}^{\prime}=j_{k}-2^{m}$. Let $\Omega_{I}$ be the sum of all such $\left[S q^{j_{1}^{\prime}}|\cdots| S q^{j_{s+1}^{\prime}}\right]$. The first proposition below together with Theorem 3.3 (ii) show that $\psi_{s}: \bar{K}_{s}(A) \otimes H^{*}\left(P^{\infty}\right) \rightarrow \bar{K}_{s+1}(A)$ defined by $\psi_{s}\left(\Gamma_{I}, \otimes x^{i}\right)=\Omega_{I}$ gives rise to the dual $\operatorname{map} \psi^{s+1}: \Lambda^{s+1} \rightarrow H_{*}\left(P^{\infty}\right) \otimes \Lambda^{s}$ in $\S 2$.

Proposition 3.5. (i) $\Omega_{I}$ is independent of the large positive integer $m$ and is an element of $\bar{K}_{s+1}(A)$.

(ii) $\Omega_{I}=\Gamma_{I}$ if $\left(i, i_{1}, \cdots, i_{s}\right)$ is an admissible sequence.

Proposition 3.6. $\psi_{*}: \bar{K}_{*}(A) \otimes H^{*}\left(P^{\infty}\right) \rightarrow \bar{K}_{*}(A)$ is a chain map.

We proceed to prove these propositions. To prove Proposition 3.5 we first begin with the case $s=1$. This case will be basis of the proof of the whole proposition and the proof of Proposition 3.6. For $s=1$ the sequence $I$ in Proposition 3.5 is $\left(i, i_{1}\right)$; so $I(m)=$ $\left(2^{m}+i, i_{1}\right)$.

Lemma 3.7. (i) $\Omega_{I}=\Omega_{\left(i, i_{1}\right)}$ is independent of the large positive integer $m$. 
(ii) $\Omega_{I}$ is equal to $\Gamma_{I}$ if $I$ is admissible and is a cycle of $\bar{K}_{2}(A)$ if $I$ is inadmissible.

In the proof of Lemma 3.7 below and other proofs later we need to determine mod 2 values of certain binomial coefficients. The following well known result on the binomal coefficients will make our computations easier.

LemMa 3.8. Let $a$ and $b$ be two nonnegative integers and let $a=\sum_{i=0}^{n} a_{i} 2^{i}, b=\sum_{i=0}^{n} b_{i} 2^{i}$ be their binary expansions where $a_{i}, b_{i}=0$ or 1 . Then

$$
\left(\begin{array}{l}
b \\
a
\end{array}\right) \equiv \prod_{i=0}^{n}\left(\begin{array}{l}
b_{i} \\
a_{i}
\end{array}\right)(\bmod 2)
$$

Proof of Lemma 3.7. We have

$$
\begin{aligned}
\Gamma_{\left\langle 2 m+i, i_{1}\right)}= & {\left[S q^{i_{1}+1} \mid S q^{2^{m}+i+1}\right] } \\
& +\sum_{j=1}^{\left[i_{1}+1+2\right]}\left(\begin{array}{l}
2^{m}+i-j \\
i_{1}+1-2 j
\end{array}\right)\left[S q^{2^{m}+i+i_{1}+2-j} \mid S q^{j}\right] .
\end{aligned}
$$

Since $i+i_{1}+2-j>0$ for $1 \leqq j \leqq\left[i_{1}+1 / 2\right]$ it follows from the construction of $\Omega_{I}$ that

$$
\Omega_{I}=\left[S q^{i_{1}+1} \mid S q^{i+1}\right]+\sum_{j=1}^{\left[i_{1}+1 / 2\right]}\left(\begin{array}{l}
2^{m}+i-j \\
i_{1}+1-2 j
\end{array}\right)\left[S q^{i+i_{1}+2-j} \mid S q^{j}\right] .
$$

By Lemma 3.8 the mod 2 value of $\left(\begin{array}{c}2^{m}+i-j \\ i_{1}+1-2 j\end{array}\right)$, for $j$ in the given range, is independent of the large positive integer $m$ (even if $i<j$ ). Thus $\Omega_{I}$ is independent of the integer $m$. This prove part (i) of the lemma.

We turn to part (ii) and we begin with the case in which $I=$ $\left(i, i_{1}\right)$ is admissible; so $2 i \geqq i_{1}$. We have

$$
\Gamma_{I}=\left[S q^{i_{1}+1} \mid S q^{i+1}\right]+\sum_{j=1}^{\left[i_{1}+1 / 2\right]}\left(\begin{array}{c}
i-j \\
i_{1}+1-2 j
\end{array}\right)\left[S q^{i_{1}+i+2-j} \mid S q^{j}\right] .
$$

$2 i \geqq i_{1}$ implies $i-j \geqq 0$ for $1 \leqq j \leqq\left[i_{1}+1 / 2\right]$; so

$$
\left(\begin{array}{l}
2^{m}+i-j \\
i_{1}+1-2 j
\end{array}\right) \equiv\left(\begin{array}{c}
i-j \\
i_{1}+1-2 j
\end{array}\right)(\bmod 2)
$$

again by Lemma 3.8. Comparing (9) and (10) we see $\Omega_{I}=\Gamma_{I}$. This proves the first half of Lemma 3.7 (ii). 
Suppose $I=\left(i, i_{1}\right)$ is inadmissible. So $2 i<i_{1}$ which implies $2(i+1) \leqq i_{1}+1$. To simplify notations let $a=i_{1}+1$ and $b=i+1$ and we rewrite (9) as

$$
\Omega_{I}=\left[S q^{a} \mid S q^{b}\right]+\sum_{j=1}^{[a / 2]}\left(\begin{array}{c}
2^{m}+b-j-1 \\
a-2 j
\end{array}\right)\left[S q^{a+b-j} \mid S q^{j}\right] .
$$

We have to show that

$$
R(a, b)=\bar{d}\left(\Omega_{I}\right)=S q^{a} S q^{b}+\sum_{j=1}^{[a / 2]}\left(\begin{array}{c}
2^{m}+b-j-1 \\
a-2 j
\end{array}\right) S q^{a+b-j} S q^{j}
$$

is zero.

The sum $R(a, b)$ can be reduced to a sum of fewer terms. For $j$ in the range $1 \leqq j \leqq b-1$ we have $b-j-1 \geqq 0$ and $(a-2 j)-$ $(b-j-1)=(a / 2-b)+(a / 2-j)+1>0$ (since $a \geqq 2 b)$; so

$$
\left(\begin{array}{c}
2^{m}+b-j-1 \\
a-2 j
\end{array}\right) \equiv 0(\bmod 2) .
$$

If $j=b$ then

$$
\left(\begin{array}{c}
2^{m}+b-j-1 \\
a-2 j
\end{array}\right)=\left(\begin{array}{c}
2^{m}-1 \\
a-2 j
\end{array}\right) \equiv 1(\bmod 2) .
$$

Thus

$$
R(a, b)=\sum_{j=b+1}^{[a / 2]}\left(\begin{array}{c}
2^{m}+b-j-1 \\
a-2 j
\end{array}\right) S q^{a+b-j} S q^{j} .
$$

We have the Adem relation

$$
\begin{aligned}
& \left(\begin{array}{c}
2^{m}+b-1 \\
a
\end{array}\right) S q^{2^{m}+a+b} \\
& \quad=S q^{a} S q^{2^{m}+b}+\sum_{j=1}^{[a / 2]}\left(\begin{array}{c}
2^{m}+b-j-1 \\
a-2 j
\end{array}\right) S q^{2^{m}+a+b-j} S q^{j} \\
& \quad=S q^{a} S q^{2^{m}+b}+S q^{2^{2 m}+a} S q^{b}+\sum_{j=b+1}^{[a \mid 2]}\left(\begin{array}{c}
2^{m}+b-j-1 \\
a-2 j
\end{array}\right) S q^{2^{2 m}+a+b-j} S q^{j} .
\end{aligned}
$$

$b-1 \geqq 0$ and $a>b-1$ imply

$$
\left(\begin{array}{c}
2^{m}+b-1 \\
a
\end{array}\right) \equiv 0(\bmod 2) .
$$

Hence 


$$
S q^{a} S q^{2^{m}+b}+S q^{2^{m}+a} S q^{b}=\sum_{j=b+1}^{[a / 2]}\left(\begin{array}{c}
2^{m}+b-j-1 \\
a-2 j
\end{array}\right) S q^{2^{m}+a+b-j} S q^{j}
$$

We shall prove $R(a, b)=0$ by relating (11) to (12).

For this purpose we will utilize Milnor multiplication to express $S q^{c} S q^{d}$ in terms of Milnor basis elements of the Steenrod algebra $A$. Let $S q(r, s)$ denote the element of $A$ dual to $\xi_{1}^{r} \xi_{2}^{s}$ in the monomial basis of the dual Hopf algebra $A^{*}=Z_{2}\left[\xi_{1}, \xi_{2}, \cdots\right]$. The rule of Milnor multiplication is given by

$$
\begin{aligned}
S q^{c} S q^{d} & =\sum_{k \geqq 0}\left(\begin{array}{c}
c+d-3 k \\
c-2 k
\end{array}\right) S q(c+d-3 k, k) \\
& =\sum_{k \geqq 0}\left(\begin{array}{c}
c+d-3 k \\
d-k
\end{array}\right) S q(c+d-3 k, k) .
\end{aligned}
$$

Applying (13) to the terms on the left side of the equation (12) we obtain

$$
S q^{a} S q^{2^{m}+b}=\sum_{k \geq 0}\left(\begin{array}{c}
2^{m}+a+b-3 k \\
2^{m}+b-k
\end{array}\right) S q\left(2^{m}+a+b-3 k, k\right)
$$

and

$$
S q^{2^{m}+a} S q^{b}=\sum_{k \geqq 0}\left(\begin{array}{c}
2^{m}+a+b-3 k \\
b-k
\end{array}\right) S q\left(2^{m}+a+b-3 k, k\right) .
$$

If $k \leqq(a+b) / 3$ and $k \leqq b$ then $\left(\begin{array}{c}2^{m}+a+b-3 k \\ 2^{m}+b-k\end{array}\right) \equiv\left(\begin{array}{c}2^{m}+a+b-3 k \\ b-k\end{array}\right)$ $(\bmod 2)$ by Lemma 3.8. If $b<k \leqq(a+b) / 3$ then both $\left(\begin{array}{c}2^{m}+a+b-3 k \\ 2^{m}+b-k\end{array}\right)$ and $\left(\begin{array}{c}2^{m}+a+b-3 k \\ b-k\end{array}\right)$ are zero $\bmod 2$. So

$$
S q^{a} S q^{2^{m}+b}+S q^{2^{m}+a} S q^{b}=\sum_{k>(a+b) / 3} c_{k} S q\left(2^{m}+a+b-3 k, k\right)
$$

where $c_{k}=0$ or 1 .

We next apply (13) to the terms in (11) and to the terms on the right side of the equation (12). We find

$$
\begin{aligned}
R(a, b) & =\sum_{j=b+1}^{[a / 2]}\left(\begin{array}{c}
2^{m}+b-j-1 \\
a-2 j
\end{array}\right)\left[\sum_{k=0}^{j}\left(\begin{array}{c}
a+b-3 k \\
j-k
\end{array}\right) S q(a+b-3 k, k)\right] \\
& =\sum_{k=0}^{[a+b / 3]}\left[\sum_{j=b+1}^{[a / 2]}\left(\begin{array}{c}
2^{m}+b-j-1 \\
a-2 j
\end{array}\right)\left(\begin{array}{c}
a+b-3 k \\
j-k
\end{array}\right)\right] S q(a+b-3 k, k)
\end{aligned}
$$

and 


$$
\begin{aligned}
& \sum_{j=b+1}^{[a / 2]}\left(\begin{array}{c}
2^{m}+b-j-1 \\
a-2 j
\end{array}\right) S q^{2^{m}+a+b-j} S q^{j} \\
& =\sum_{j=b+1}^{[a / 2]}\left(\begin{array}{c}
2^{m}+b-j-1 \\
a-2 j
\end{array}\right)\left[\sum_{k=0}^{j}\left(\begin{array}{c}
2^{m}+a+b-3 k \\
j-k
\end{array}\right) S q\left(2^{m}+a+b-3 k, k\right)\right] \\
& =\sum_{k=0}^{[a+b / 3]}\left[\sum_{j=b+1}^{[a / 2]}\left(\begin{array}{c}
2^{m}+b-j-1 \\
a-2 j
\end{array}\right)\left(\begin{array}{c}
2^{m}+a+b-3 k \\
j-k
\end{array}\right)\right] S q\left(2^{m}+a+b-3 k, k\right) \\
& +\sum_{k>(a+b) / 3}\left[\sum_{j=b+1}^{[a / 2]}\left(\begin{array}{c}
2^{m}+b-j-1 \\
a-2 j
\end{array}\right)\left(\begin{array}{c}
2^{m}+a+b-3 k \\
j-k
\end{array}\right)\right] \\
& \times S q\left(2^{m}+a+b-3 k, k\right) .
\end{aligned}
$$

Since $S q\left(2^{m}+a+b-3 k, k\right)$ are linearly independent over $Z_{2}$ it follows from (12), (14) and (16) that

$$
\sum_{j=b+1}^{[a / 2]}\left(\begin{array}{c}
2^{m}+b-j-1 \\
a-2 j
\end{array}\right)\left(\begin{array}{c}
2^{m}+a+b-3 k \\
j-k
\end{array}\right) \equiv 0(\bmod 2)
$$

for $k$ in the range $0 \leqq k \leqq(a+b) / 3$. Since $m$ is large we have that $\left(\begin{array}{c}2^{m}+a+b-3 k \\ j-k\end{array}\right) \equiv\left(\begin{array}{c}a+b-3 k \\ j-k\end{array}\right)(\bmod 2)$ for $k$ in the same range. So

$$
\sum_{j=b+1}^{[a / 2]}\left(\begin{array}{c}
2^{m}+b-j-1 \\
a-2 j
\end{array}\right)\left(\begin{array}{c}
a+b-3 k \\
j-k
\end{array}\right) \equiv 0(\bmod 2)
$$

for $0 \leqq k \leqq(a+b) / 3$. Thus from (15) we see $R(a, b)=0$. This completes the proof of Lemma 3.7 (ii).

The following lemma, which will be needed in the proofs of Proposition 3.5 and Proposition 3.6, is immediate from the structure of $\bar{K}_{*}(A)$.

LEMMA 3.9. Let $R=\sum_{j}\left[S q^{j_{1}}|\cdots| S q^{j_{s}}\right]$ be an element of $\bar{K}_{s}(A)(s \geqq 2)$ and let $\left\{I_{\nu}=\left(i_{1}(\nu), \cdots, i_{s}(\nu)\right\}_{\nu=1}^{n}\right.$ be the set of all admissible sequences such that $\left[S q^{i_{s}(\nu)+1}|\cdots| S q^{i_{1}(\nu)+1}\right] \in R$. Then $R=\sum_{\nu=1}^{n} \Gamma_{I_{\nu}}$.

Proof of Proposition 3.5. The case $s=1$ is Lemma 3.7; so we assume $s>1$. We begin with part (i). In part (i) there are two conclusions, namely

(a) $\Omega_{I}$ is independent of the large positive integer $m$ and

(b) $\Omega_{I}$ is an element of $\bar{K}_{s+1}(A)$.

We shall prove (a) and (b) simultaneously. For this purpose we restate these two results in the following manner. Recall that $\Omega_{I}$ is constructed from $\Gamma_{I(m)}$ where $I(m)=\left(2^{m}+i, i_{1}, \cdots, i_{s}\right)$; so we should write $\Omega_{I}$ as $\Omega_{I(m)}$ to indicate that it depends on $m$. We fix 
a large positive integer $m$. Then (a) and (b) together are equivalent to

and

(c) $\Omega_{I(m)}$ is an element of $\bar{K}_{\mathrm{s}+1}(A)$

(d) $\Omega_{I(m)}=\Omega_{I\left(m^{\prime}\right)}$ for any other large positive integer $m^{\prime}$.

We shall verify (c) and (d) by proving (c) and a result which implies (d). To describe this result let $m^{\prime}$ be as in (d). We construct an element $\bar{\Gamma}_{I\left(m^{\prime}\right)} \in \bar{B}_{s+1}(A)$ as follows. For each $\left[S q^{j_{1}}|\cdots| S q^{j_{s+1}}\right] \in$ $\Gamma_{I(m)}$ let $j_{k}$ be the only integer such that $j_{k}>2^{m}$ and consider $\left[S q^{j_{1}^{\prime \prime}}|\cdots| S q_{s+1}^{j^{\prime \prime}}\right]$ where $j_{l}^{\prime \prime}=j_{l}$ if $l \neq k$ and $j_{k}^{\prime \prime}=j_{k}-2^{m}+2^{m^{\prime}}$. Let $\bar{\Gamma}_{I\left(m^{\prime}\right)}$ be the sum of all such $\left[S q^{j_{1}^{\prime \prime}}|\cdots| S q_{s+1}^{j_{s+1}^{\prime \prime}}\right]$. We claim

(e) $\bar{\Gamma}_{I\left(m^{\prime}\right)}=\Gamma_{I\left(m^{\prime}\right)}$.

It is clear that (d) follows from (e). Note that $\left(2^{m^{\prime}}+i, i_{1}, \cdots, i_{s}\right)$ is the only admissible sequence such that

$$
\left[S q^{i_{s}+1}|\cdots| S q^{i_{1}+1} \mid S q^{2^{m}+i+1}\right] \in \bar{\Gamma}_{I\left(m^{\prime}\right)} .
$$

So by Lemma 3.9 (e) is equivalent to

(e) $\bar{\Gamma}_{I\left(m^{\prime}\right)}$ is an element of $\bar{K}_{s+1}(A)$.

We proceed to prove (c) and (e)' and we prove them by using Theorem 3.3 (iii) and Remark 3.4. Suppose $\Gamma_{I(m)}=\sum_{j}\left[S q^{j_{1}}|\cdots| S q^{j_{s+1}}\right]$. Let $\Omega_{I(m)}=\sum_{j}\left[S q_{1}^{j_{1}^{\prime}}|\cdots| S q_{s+1}^{j_{s}^{\prime}}\right]$ and $\bar{\Gamma}_{I\left(m^{\prime}\right)}=\sum_{j}\left[S q^{j_{1}^{\prime \prime}}|\cdots| S q^{j_{s+1}^{\prime \prime}}\right]$ be the corresponding sums. For each $r(1 \leqq r \leqq s)$ and each pair of sequences $\left(k_{1}, \cdots, k_{r-1}\right)$ and $\left(k_{r+2}, \cdots, k_{s+1}\right)$ consider the sums

$$
\begin{aligned}
& B=\sum_{j}\left[S q^{j_{r}} \mid S q^{j_{r+1}}\right], \\
& C=\sum_{j}\left[S q^{j_{r}^{\prime}} \mid S q^{j_{r+1}^{\prime}}\right]
\end{aligned}
$$

and

$$
D=\sum_{j}\left[S q_{r}^{j_{r}^{\prime \prime}} \mid S q_{r+1}^{\prime \prime}\right]
$$

where the summation in $B$ is taken over all $j$ such that $\left(j_{1}, \cdots, j_{r-1}\right)=$ $\left(k_{1}, \cdots, k_{r-1}\right),\left(j_{r+2}, \cdots, j_{s+1}\right)=\left(k_{r+2}, \cdots, k_{s+1}\right)$ and the summations in $C$ and $D$ are taken, respectively, over all $j$ such that $\left(j_{1}^{\prime}, \cdots, j_{r-1}^{\prime}\right)=$ $\left(k_{1}^{\prime}, \cdots, k_{r-1}^{\prime}\right),\left(j_{r+2}^{\prime}, \cdots, j_{s+1}^{\prime}\right)=\left(k_{r+2}^{\prime}, \cdots, k_{s+1}^{\prime}\right),\left(j_{1}^{\prime \prime}, \cdots, j_{r-1}^{\prime \prime}\right)=\left(k_{1}^{\prime \prime}, \cdots, k_{r-1}^{\prime \prime}\right)$ and $\left(j_{r+2}^{\prime \prime}, \cdots, j_{s+1}^{\prime \prime}\right)=\left(k_{r+2}^{\prime \prime}, \cdots, k_{s+1}^{\prime \prime}\right)$. By Theorem 3.3 (iii) and Remark $3.4 B$ is an element of $\bar{K}_{2}(A)$. We have to show that $C$ and $D$ are elements of $\bar{K}_{2}(A)$.

If $k_{q}>2^{m}$ for some $q$ then $j_{r}=j_{r}^{\prime}=j_{r}^{\prime \prime}$ and $j_{r+1}=j_{r+1}^{\prime}=j_{r+1}^{\prime \prime}$ for each $\left[S q_{r}^{j_{r}^{\prime}} \mid S q_{r+1}^{\prime}\right]$ in the sum $C$ and each $\left[S q_{r}^{j \prime \prime} \mid S q_{r+1}^{\prime \prime}\right]$ in the sum $D$. So $C=D=B$ which is an element of $\bar{K}_{2}(A)$.

If $k_{q}<2^{m}$ for all $q$ then $k_{q}^{\prime}=k_{q}^{\prime \prime}=k_{q}$ all $q$ and 


$$
\begin{aligned}
B & =\sum_{\mu=1}^{n} \Gamma\left(2^{m}+a_{\mu}, b_{\mu}\right) \\
& =\sum_{\mu=1}^{n}\left[S q^{b_{\mu}+1} \mid S q^{2^{m}+a_{\mu}+1}\right]+\sum_{j=1}^{\left[b_{\mu}+1 / 2\right]}\left(\begin{array}{l}
2^{m}+a_{\mu}-j \\
b_{\mu}+1-2 j
\end{array}\right)\left[S q^{2^{m}+a_{\mu}+b_{\mu}+2-j} \mid S q^{j}\right] .
\end{aligned}
$$

From the constructions of $\Omega_{I(m)}$ and $\bar{\Gamma}_{I\left(m^{\prime}\right)}$ we have

$$
C=\sum_{\mu=1}^{n} \Omega_{\left(a_{\mu}, b_{\mu}\right)}
$$

and

$$
\begin{aligned}
& D=\sum_{\mu=1}^{n}\left[S q^{b_{\mu}+1} \mid S q^{2^{m^{\prime}+a_{\mu}+1}}\right] \\
& +{ }^{\left[b_{\mu}+1^{\prime} \cdot 2\right]}\left(\begin{array}{l}
2^{m}+a_{\mu}-j \\
b_{\mu}+1-2 j
\end{array}\right)\left[S q^{2^{m^{\prime}}+a_{\mu}+b_{\mu}+2-j} \mid S q^{j}\right] .
\end{aligned}
$$

By Lemma 3.7 each $\Omega_{\left(a_{\mu}, b_{\mu}\right)}$ is an element of $\bar{K}_{2}(A)$; so $C \in \bar{K}_{2}(A)$. Since $m$ and $m^{\prime}$ are large it follows from Lemma 3.8 that

$$
\left(\begin{array}{l}
2^{m}+a_{\mu}-j \\
b_{\mu}+1-2 j
\end{array}\right) \equiv\left(\begin{array}{c}
2^{m^{\prime}}+a_{\mu}-j \\
b_{\mu}+1-2 j
\end{array}\right)(\bmod 2)
$$

for $1 \leqq j \leqq\left[b_{\mu}+1 / 2\right]$. Replacing $m$ in (17) by $m^{\prime}$ we see that $D=\sum_{\mu=1}^{n} \Gamma_{\left(2^{m^{\prime}}+a_{\mu}, b_{\mu}\right)}$ which belongs to $\bar{K}_{2}(A)$. This proves (c) and (e) and therefore part (i) of Proposition 3.5.

We turn to part (ii); so we assume $I=\left(i, i_{1}, \cdots, i_{\mathrm{s}}\right)$ is admissible. Suppose $m \gg i+i_{1}+\cdots+i_{s}+s$. It is easy to see that except for $\left(i, i_{1}, \cdots, i_{s}\right)$ there is no other admissible sequence $\left(j_{1}, \cdots, j_{s+1}\right)$ such that

$$
\lambda_{2^{m}+i_{1}} \cdots \lambda_{i_{s}} \in \lambda_{2^{m}{ }_{j_{1}}} \lambda_{j_{2}} \cdots \lambda_{j_{s+1}}
$$

By Lemma 3.2 (ii)

$$
\lambda_{2}{ }_{+i} \lambda_{i} \lambda_{i_{1}} \cdots \lambda_{i_{s}} \notin \lambda_{l_{1}} \cdots \lambda_{l_{k-1}} \lambda_{2^{m}+l_{k}} \lambda_{l_{k+1}} \cdots \lambda_{l_{s+1}}
$$

for any admissible sequence $\left(l_{1}, \cdots, l_{s+1}\right)$ and any integer $k$ in the range $2 \leqq k \leqq s+1$. It follows, then, that $\left(i, i_{1}, \cdots, i_{s}\right)$ is the only admissible sequence such that $\left[S q^{i_{s}+1}|\cdots| S q^{i_{1}+1} \mid S q^{i+1}\right] \in \Omega_{I}$. Since $\Omega_{I} \in \bar{K}_{s+1}(A)$, by Lemma 3.9 , we see that $\Omega_{I}=\Gamma_{I}$. This proves Proposition 3.5 (ii).

Proof of Proposition 3.6. We have to show that for each $s \geqq 0$ the square 


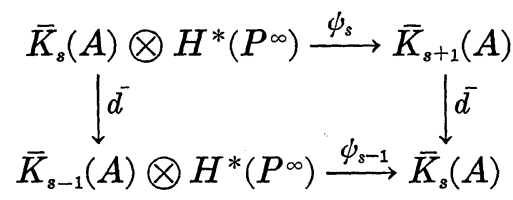

is commutative. The result is obviously true for $s=0$. For $s=1$ consider any basis element $S q^{i_{1}+1} \otimes x^{i}$ of $\bar{K}_{1}(A) \otimes H^{*}\left(P^{\infty}\right)$. We have $\psi_{1}\left(S q^{i_{1}+1} \otimes x^{i}\right)=\Omega_{\left(i, i_{1}\right)}$. If $\left(i, i_{1}\right)$ is admissible then, by Lemma 3.7 , $\Omega_{\left(i, i_{1}\right)}=\Gamma_{\left(i, i_{1}\right)}$; so from formula (8) we have

$$
\left(\bar{d} \psi_{1}\right)\left(S q^{i_{1}+1} \otimes x^{i}\right)=\bar{d}\left(\Omega_{\left(i, i_{1}\right)}\right)=\left(\begin{array}{c}
i \\
i_{1}+1
\end{array}\right) S q^{i+i_{1}+2}
$$

This equation is still true if $\left(i, i_{1}\right)$ is inadmissible because, then, $\bar{d}\left(\Omega_{\left(i, i_{1}\right)}\right)=0$ by Lemma 3.7 (ii) and $2 i<i_{1}$ implies $i_{1}+1>i$ which in turn implies $\left(\begin{array}{c}i \\ i_{1}+1\end{array}\right) \equiv 0(\bmod 2)$. On the other way of square (18) we find

$$
\begin{aligned}
\left(\psi_{0} \bar{d}\right)\left(S q^{i_{1}+1} \otimes x^{i}\right) & =\psi_{0}\left(\left(\begin{array}{c}
i \\
i_{1}+1
\end{array}\right) 1 \otimes x^{i+i_{1}+1}\right) \\
& =\left(\begin{array}{c}
i \\
i_{1}+1
\end{array}\right) S q^{i+i_{1}+2}
\end{aligned}
$$

Thus the result is true for $s=1$.

Suppose $s>1$. Consider any basis element $\Gamma_{I^{\prime}} \otimes x^{i}$ of $\bar{K}_{s}(A) \otimes$ $H^{*}\left(P^{\infty}\right)$ where $I^{\prime}=\left(i_{1}, \cdots, i_{s}\right)$. We have $\psi_{s}\left(\Gamma_{I^{\prime}} \otimes x^{\prime}\right)=\Omega_{I}$ where $I=\left(i, i_{1}, \cdots, i_{s}\right)$. We shall prove $\left(\bar{d} \psi_{s}\right)\left(\Gamma_{I^{\prime}} \otimes x^{i}\right)=\left(\psi_{s-1} \bar{d}\right)\left(\Gamma_{I^{\prime}} \otimes x^{i}\right)$ by showing that the maps

$$
\begin{aligned}
& \Gamma_{I^{\prime}} \otimes x^{i} \longrightarrow \Gamma_{I^{\prime}} \otimes x^{2^{m}+i}, \\
& \Gamma_{I^{\prime}} \otimes x^{2^{m}+i} \longrightarrow \Gamma_{I(m)}
\end{aligned}
$$

and

$$
\Gamma_{I(m)} \longrightarrow \Omega_{I}
$$

commute with boundary homomorphisms where $m \gg i+i_{1}+\cdots+i_{s}+s$ and $I(m)=\left(2^{m}+i, i_{1}, \cdots, i_{s}\right)$. We denote these maps by $f_{1}, f_{2}$ and $f_{3}$ in that order.

That $f_{1}$ commutes with boundary homomorphisms is easy to verify; we just notice that $S q^{k} x^{i}=\left(\begin{array}{l}i \\ k\end{array}\right) x^{i+k}, S q^{k} x^{2^{m}+i}=\left(\begin{array}{c}2^{m}+i \\ k\end{array}\right) x^{2^{m}+i+k}$ and $\left(\begin{array}{c}2^{m}+i \\ k\end{array}\right) \equiv\left(\begin{array}{l}i \\ k\end{array}\right)(\bmod 2)$ for $k<i_{1}+\cdots+i_{s}$. 
To prove that $f_{2}$ commutes with boundary homomorphisms is to show

$$
f_{2}\left(\bar{d}\left(\Gamma_{I^{\prime}} \otimes x^{2^{m}+i}\right)\right)=\bar{d}\left(\Gamma_{I(m)}\right) .
$$

By Theorem 3.3 (iii) for each integer $\nu \geqq 1$ the sum $T^{\prime \prime}(\nu)$ of all $\left[S q^{j_{1}}|\cdots| S q^{j_{s-1}}\right]$ with $\left[S q^{j_{1}}|\cdots| S q^{j_{s-1}} \mid S q^{\cdot}\right] \in \Gamma_{I^{\prime}}$ is an element of $\bar{K}_{s-1}(A)$. We write $\left[T^{\prime \prime}(\nu) \mid S q^{2}\right]$ to denote the sum of all $\left[S q^{j_{1}}|\cdots| S q^{j_{s-1}} \mid S q^{\nu}\right]$ such that $\left[S q^{j_{1}}|\cdots| S q^{j_{s-1}}\right] \in T^{\prime \prime}(\nu)$. So $\Gamma_{I^{\prime}}=\sum_{\nu \geqq 1}\left[T^{\prime \prime}(\nu) \mid S q^{\nu}\right]$ which is a finite sum since $T^{\prime \prime}(\nu)=0$ for almost all integers $\nu$. Then

$$
\bar{d}\left(\Gamma_{I^{\prime}} \otimes x^{2^{m}+i}\right)=\bar{d}\left(\Gamma_{I^{\prime}}\right) \otimes x^{2^{m}+i}+\sum_{\nu \geqq 1}\left(\begin{array}{c}
2^{m}+i \\
\nu
\end{array}\right) T^{\prime \prime}(\nu) \otimes x^{2^{m}+\imath+\nu} .
$$

Let $\bar{d}\left(\Gamma_{I^{\prime}}\right)=\sum_{l} \Gamma_{I^{\prime \prime}(l)}$ and let $T^{\prime \prime}(\nu)=\sum_{k_{\nu}} \Gamma_{I^{\prime \prime}\left(\nu, k_{\nu}\right)}$. Consider the admissible sequences $I^{\prime}(l, m)=\left(2^{m}+i, I^{\prime \prime}(l)\right)$ (i.e., $I^{\prime}(l, m)=\left(2^{m}+i\right.$, $\left.i_{1}(l), \cdots, i_{s-1}(l)\right)$ if $\left.I^{\prime \prime}(l)=\left(i_{1}(l), \cdots, i_{s-1}(l)\right)\right)$ and $I^{\prime}\left(\nu, k_{\nu}, m\right)=\left(2^{m}+i+\nu\right.$, $\left.I^{\prime \prime}\left(\nu, k_{\nu}\right)\right)$. Let $\left.T^{\prime}(\nu, m)=\sum_{k_{\nu}} \Gamma_{I^{\prime}\left(\nu, k_{\nu}, m\right.}\right)$. Then

$$
f_{2}\left(\bar{d}\left(\Gamma_{I^{\prime}} \otimes x^{2^{m}+i}\right)\right)=\sum_{l} \Gamma_{I^{\prime}(l, m)}+\sum_{\nu \geqq 1}\left(\begin{array}{c}
2^{m}+i \\
\nu
\end{array}\right) T^{\prime}(\nu, m) .
$$

It remains to show that $\bar{d}\left(\Gamma_{I(m)}\right)$ is equal to the right side of the above equation.

It is easy to see that $\lambda_{i_{1}} \cdots \lambda_{i_{s}} \in \lambda_{j_{1}} \cdots \lambda_{j_{s}}$ if and only if $\lambda_{2^{m}+i} \lambda_{i_{1}} \cdots \lambda_{i_{s}} \in \lambda_{2^{m}+i} \lambda_{j_{1}} \cdots \lambda_{j_{s}}$ and that $\lambda_{2^{m}+i} \lambda_{i_{1}} \cdots \lambda_{i_{s}} \notin \lambda_{k_{0}} \lambda_{k_{1}} \cdots \lambda_{k_{s}}$ for any sequence $\left(k_{0}, k_{1}, \cdots, k_{s}\right)$ such that $k_{0}>2^{m}, k_{0} \neq 2^{m}+i$ and $k_{0}+$ $k_{1}+\cdots+k_{s}=2^{m}+i+i_{1}+\cdots+i_{s}$. Hence $\left[\Gamma_{I^{\prime}} \mid S q^{2^{m}+i+1}\right]$ is a subsum of $\Gamma_{I(m)}$ and for each $\left[S q^{j_{1}}|\cdots| S q^{j_{s+1}}\right] \in D_{I(m)} \equiv \Gamma_{I(m)}-\left[\Gamma_{I^{\prime}} \mid S q^{2^{m}+i+1}\right]$ the integer $r$ with $j_{r}>2^{m}$ is less than $s+1$. We have

$$
\begin{aligned}
\Gamma_{I(m)} & =\left[\Gamma_{I^{\prime}} \mid S q^{2^{m}+i+1}\right]+D_{I(m)} \\
& =\sum_{\nu \geqq 1}\left[T^{\prime \prime}(\nu)\left|S q^{\nu}\right| S q^{2{ }^{2}+i+1}\right]+D_{I(m)} .
\end{aligned}
$$

By Theorem 3.3 (iii) and Remark 3.4 we see that there is a subsum $L$ of $D_{I(m)}$ such that

$$
\sum_{\nu \geq 1}\left[T^{\prime \prime}(\nu)\left|S q^{\nu}\right| S q^{2^{m}+i+1}\right]+L=\sum_{\nu \geq 1}\left[T^{\prime \prime}(\nu) \mid \Gamma_{\left(2^{m}+i, \nu-1\right)}\right]
$$

where for $\theta_{1} \in \bar{K}_{s-1}(A)$ and $\theta_{2} \in \bar{K}_{2}(A)\left[\theta_{1} \mid \theta_{2}\right]$ denotes the sum of all $\left[S q^{j_{1}}|\cdots| S q^{j_{s-1}}\left|S q^{k_{1}}\right| S q^{k_{2}}\right]$ such that $\left[S q^{j_{1}}|\cdots| S q^{j_{s-1}}\right] \in \theta_{1}$ and $\left[S q^{k_{1}} \mid S q^{k_{2}}\right] \in$ $\theta_{2}$. It is easy to see that $L$ is precisely the sum of all $\left[S q^{j_{1}}|\cdots| S q^{j_{s}} \mid S q^{j_{s+1}}\right] \in D_{I(u)}$ such that $j_{s}>2^{m}$; so for each $\left[S q^{j_{1}} \cdots S q^{j_{s+1}}\right] €$ $M=D_{I(m)}-L$ the integer $r$ with $j_{r}>2^{m}$ is less than $s$. Then 


$$
\begin{aligned}
\bar{d}\left(\Gamma_{I(m)}\right)= & \bar{d}\left(\left[\Gamma_{I^{\prime}} \mid S q^{2^{m}+i+1}\right]+D_{I(m)}\right) \\
= & \bar{d}\left(\sum_{\nu \geq 1}\left[T^{\prime \prime}(\nu)\left|S q^{\nu}\right| S q^{2^{m}+i+1}\right]+L+M\right) \\
= & {\left[\bar{d}\left(\Gamma_{I^{\prime}}\right) \mid S q^{2^{m}+i+1}\right]+\sum_{\nu \geq 1}\left[T^{\prime \prime}(\nu) \mid \bar{d}\left(\Gamma_{\left(2^{m+i, \nu-1)}\right)}\right]+N\right.} \\
= & \sum_{l}\left[\Gamma_{I^{\prime \prime}(l)} \mid S q^{2^{m}+i+1}\right] \\
& +\sum_{\nu \geqq 1}\left(\begin{array}{c}
2^{m}+i \\
\nu
\end{array}\right)\left[\sum_{k_{\nu}} \Gamma_{I^{\prime \prime}\left(\nu, k_{\nu}\right)} \mid S q^{2^{m}+i+\nu+1}\right]+N
\end{aligned}
$$

where each sequence $\left(j_{1}, \cdots, j_{s}\right)$ with $\left[S q^{j_{s}+1}|\cdots| S q^{j_{1}+1}\right] \in N$ is inadmissible. The set of admissible sequences $\left(j_{1}, \cdots, j_{s}\right)$ with

$$
\begin{aligned}
& {\left[S q^{j_{s}+1}|\cdots| S q^{j_{1}+1}\right] \in \sum_{l}\left[\Gamma_{I^{\prime \prime}(l)} \mid S q^{2^{m}+i+1}\right]} \\
& \quad+\sum_{\nu \geq 1}\left(\begin{array}{c}
2^{m}+i \\
\nu
\end{array}\right)\left[\sum_{k} \Gamma_{I^{\prime \prime}\left(\nu, k_{\nu}\right)} \mid S q^{2^{m}+i+\nu+1}\right]
\end{aligned}
$$

is equal to the set of admissible sequences $\left(j_{1}^{\prime}, \cdots, j_{s}^{\prime}\right)$ with

$$
\left[S q^{j_{s}^{\prime}+1}|\cdots| S q^{j_{1}^{\prime+1}}\right] \in \sum_{l} \Gamma_{I^{\prime}(l, m)}+\sum_{\nu \geq 1}\left(\begin{array}{c}
2^{m}+i \\
\nu
\end{array}\right) T^{\prime}(\nu, m) .
$$

So by Lemma 3.9 we have

$$
\bar{d}\left(\Gamma_{I(m)}\right)=\sum_{l} \Gamma_{I^{\prime}(l, m)}+\sum_{\nu \geqq 1}\left(\begin{array}{c}
2^{m}+i \\
\nu
\end{array}\right) T^{\prime}(\nu, m)=f_{2}\left(\bar{d}\left(\Gamma_{I^{\prime}} \otimes x^{2^{m}+i}\right)\right) .
$$

This proves that $f_{2}$ commutes with $\bar{d}$.

Finally we prove that $f_{3}$ commutes with $\bar{d}$. Let

$$
\Gamma_{I(m)}=\sum_{j}\left[S q^{j_{1}}|\cdots| S q^{j_{s+1}}\right]
$$

and let $f_{3}\left(\Gamma_{I(m)}\right)=\Omega_{I}=\sum_{j}\left[S q^{j_{1}^{\prime}}|\cdots| S q^{j_{s+1}^{\prime}}\right]$ be the corresponding sum. For each $r(1 \leqq r \leqq s)$ and each pair of sequences $K_{1}=\left(k_{1}, \cdots, k_{r-1}\right)$ and $K_{2}=\left(k_{r+2}, \cdots, k_{s+1}\right)$ the sums

$$
U=\sum_{j}\left[S q^{j_{r}} \mid S q^{j_{r+1}}\right]
$$

and

$$
V=\sum_{j}\left[S q_{r}^{j_{r}^{\prime}} \mid S q^{j_{r+1}^{\prime}}\right]
$$

are elements of $\bar{K}_{2}(A)$ by Theorem 3.3 (iii) and Remark 3.4 where the summation in $U$ is taken over all $j$ such that $\left(j_{1}, \cdots, j_{r-1}\right)=$ $\left(k_{1}, \cdots, k_{r-1}\right), \quad\left(j_{r+2}, \cdots, j_{s+1}\right)=\left(k_{r+2}, \cdots, k_{s+1}\right)$ and the summation in $V$ is taken over all $j$ such that $\left(j_{1}^{\prime}, \cdots, j_{r-1}^{\prime}\right)=\left(k_{1}^{\prime}, \cdots, k_{r-1}^{\prime}\right)$ and $\left(j_{r+2}^{\prime}, \cdots, j_{s+1}^{\prime}\right)=\left(k_{r+2}^{\prime}, \cdots, k_{s+1}^{\prime}\right)$. Let 


$$
U\left(K_{1}, K_{2}\right)=\left[S q^{k_{1}}|\cdots| S q^{k_{r-1}}|\bar{d}(U)| S q^{k_{r+2}}|\cdots| S q^{k_{s+1}}\right]
$$

and

$$
V\left(K_{1}, K_{2}\right)=\left[S q_{1}^{k_{1}^{\prime}}|\cdots| S q_{r-1}^{k^{\prime}}|\bar{d}(V)| S q_{r+2}^{k}|\cdots| S q_{s+1}^{k \prime}\right] .
$$

The sum of all elements $U\left(K_{1}, K_{2}\right)$ is equal to $\bar{d}\left(\Gamma_{I(m)}\right)$ and the sum of all elements $V\left(K_{1}, K_{2}\right)$ is equal to $\bar{d}\left(\Omega_{I}\right)$; furthermore, $U\left(K_{1}, K_{2}\right) \rightarrow$ $V\left(K_{1}, K_{2}\right)$ is a one-to-one correspondence between these two sets of elements. We shall prove $f_{3}\left(\bar{d}\left(\Gamma_{I(m)}\right)\right)=\bar{d}\left(\Omega_{I}\right)=\bar{d}\left(f_{3}\left(\Gamma_{I(m)}\right)\right)$ by showing that, under $f_{3}$, each $U\left(K_{1}, K_{2}\right)$ goes to the corresponding $V\left(K_{1}, K_{2}\right)$.

Suppose $K_{1}, K_{2}, U$ and $V$ are as given above. We need to discuss $\bar{d}(U)$ and $\bar{d}(V)$ and we do this in two cases. The first case is that $k_{q}>2^{m}$ for some $q$. In this case $k_{q}^{\prime}=k_{q}-2^{m}, k_{l}^{\prime}=k_{l}$ if $l \neq q, \quad j_{r}^{\prime}=j_{r}$ and $j_{r+1}^{\prime}=j_{r+1}$ for all $\left[S q_{r}^{j_{r}^{\prime}} \mid S q^{j_{r+1}^{\prime}}\right] \in V$. So $U=V$. Since $\bar{d}(U)=\bar{d}(V)=S q^{c}$ or 0 and $c<2^{m}$ it follows from the definition of $f_{3}$ that $U\left(K_{1}, K_{2}\right)$ goes to $V\left(K_{1}, K_{2}\right)$ under $f_{3}$.

The other case is that $k_{l}<2^{m}$ for all $l$; so $k_{l}^{\prime}=k_{l}$ all $l$. Then

$$
U=\sum_{\mu=1}^{n} \Gamma_{\left(2^{\left.m+a_{\mu}, b_{\mu}\right)}\right.} \text { and so } V=\sum_{\mu=1}^{n} \Omega_{\left(a_{\mu}, b_{\mu}\right)} \text {. }
$$

By formulae (8) and (19) we have

$$
\bar{d}(U)=\sum_{\mu=1}^{n} \bar{d}\left(\Gamma_{\left(2^{m}+a_{\mu}, b_{\mu}\right)}\right)=\sum_{\mu=1}^{n}\left(\begin{array}{c}
2^{m}+a_{\mu} \\
b_{\mu}+1
\end{array}\right) S q^{2^{m}+a_{\mu}+b_{\mu}+2}
$$

and

$$
\bar{d}(V)=\sum_{\mu=1}^{n} \bar{d}\left(\Omega_{\left(a_{\mu}, b_{\mu}\right)}\right)=\sum_{\mu=1}^{n}\left(\begin{array}{c}
a_{\mu} \\
b_{\mu}+1
\end{array}\right) S q^{a^{\mu}+b_{\mu}+2}
$$

So

$$
U\left(K_{1}, K_{2}\right)=\sum_{\mu=1}^{n}\left(\begin{array}{c}
2^{m}+a_{\mu} \\
b_{\mu}+1
\end{array}\right)\left[S q^{k_{1}}|\cdots| S q^{k_{r-1}}\left|S q^{2^{m}+a_{\mu}+b_{\mu}+2}\right| S q^{k_{r+2}}|\cdots| S q^{k_{s+1}}\right]
$$

and

$$
V\left(K_{1}, K_{2}\right)=\sum_{\mu=1}^{n}\left(\begin{array}{c}
a_{\mu} \\
b_{\mu}+1
\end{array}\right)\left[S q^{k_{1}}|\cdots| S q^{k_{r-1}}\left|S q^{a_{\mu}+b_{\mu}+2}\right| S q^{k_{r+2}}|\cdots| S q^{k_{s+1}}\right]
$$

Since $m$ is large, $\left(\begin{array}{c}2^{m}+a_{\mu} \\ b_{\mu}+1\end{array}\right) \equiv\left(\begin{array}{c}a_{\mu} \\ b_{\mu}+1\end{array}\right)(\bmod 2)$ for all $\mu$. Thus $U\left(K_{1}, K_{2}\right)$ goes to $V\left(K_{1}, K_{2}\right)$ under $f_{3}$. This completes the proof of Proposition 3.6 and therefore the proof of Proposition 2.1 (i). 


\section{REFERENCES}

1. E. B. Curtis, Simplicial homotopy theory, Advances in Math., 6 (1971), 107-207.

2. D. S. Kahn and S. B. Priddy, Applications of the transfer to stable homotopy theory, Bull. Amer. Math. Soc., 78 (1972), 981-991.

3. M. E. Mahowald, The metastable homotopy of $S^{n}$, Memo. Amer. Math. Soc., 72 (1967).

4. S. B. Priddy, Koszul resolutions, Trans. Amer. Math. Soc., 152 (1970), 39-60.

5. G. W. Whitehead, Recent Advances in Homotopy Theory, Regional Conference Series in Mathematics, No. 5 (1970).

Received July 24, 1979. Partially supported by National Science council of the Republic of China.

National Tsing Hua University

Hsinchu, TaIwan, Republic of China 
